\title{
Imitation et désacralisation de Claudel au Canada français
}

\author{
Bernard Hue \\ Université de Haute-Bretagne, Rennes II
}

On a pu dire que Claudel avait connu un véritable culte au Canada au lendemain de ses visites effectuées à l'automne de 1928 et de $1929^{1}$. Accueilli avec force honneurs, l'ambassadeur, alors en poste aux Etats-Unis, suscite chez les Canadiens, ces " chers frères lointains ", ses "frères Canadiens " ${ }^{2}$, un véritable engouement dans les provinces francophones, demeurées sous l'emprise de la tradition issue de l'époque de la colonisation française, elle-même dominée par l'église catholique, et perpétuée jusqu'à la Révolution tranquille. Claudel, on le conçoit, ne pouvait que se réjouir d'être l'invité des universités catholiques de Montréal, Québec, Toronto. Il exprimera encore sa satisfaction, en 1937, en évoquant " cette nouvelle France ${ }^{3}$ qui, outre la langue, a la chance d'avoir comme assise fondamentale la religion catholique. De ses séjours, des représentations théâtrales données ici et là à cette occasion, il devait laisser plus qu'un simple souvenir 4. Si son théâtre paraît d'accès difficile, beaucoup s'enthousiasment pour son œuvre poétique. Il devient, sans conteste, le grand auteur à la mode, le plus représentatif de la littérature religieuse, le modèle destiné, peut-on penser, à faire naître des imitations, voire des vocations littéraires. Il serait donc intéressant de dresser un bilan, même approximatif, permettant d'apprécier l'effet de ce modèle accueilli avec enthousiasme et de se demander si celui-ci était appelé à durer, compte tenu de l'évolution qui, peu à peu, allait engager toute une société figée dans un immobilisme socioculturel apparemment inébranlable, à caractère fortement clérical, dans une révolution dite tranquille. 
Comme le rappelle Maurice Lebel, le souvenir des événements correspondant aux visites de Claudel devait rester très vivant et produire des effets non négligeables dans la production littéraire canadienne française, ainsi que dans l'enseignement, les Universités inscrivant à leurs programmes de cours et de recherches différentes œuvres de Claudel, malgré l'aspect ardu que leur confère une certaine obscurité liée à l'héritage symboliste et à la leçon de Rimbaud. Le même critique fait état de l'engouement durable des critiques, des professeurs, des chercheurs, des écrivains, de la ferveur engendrés par Claudel et parle d'un mythe relayé par la radio et la télévision.

Dans la perspective de l'attrait exercé par l'œuvre de Claudel et de son éventuelle influence sur la production littéraire du Canada francophone, à supposer que ce poète, auteur catholique souvent considéré comme hermétique, pût avoir des émules non réduits à de simples plagiaires, Maurice Lebel pose judicieusement le problème en ces termes : «Il conviendrait qu'on analyse l'apport de la poésie chrétienne de Claudel à la poésie religieuse du Canada français » et met ainsi l'accent sur la nature de l'œuvre en question. Selon lui, même limité à la poésie, l'apport claudélien serait considérable. "À commencer, par exemple », dit-il, " par Gustave Lamarche, membre de l'Académie canadienne-française ${ }^{5}$. "

Cet exemple, révélateur de l'enthousiasme suscité par le modèle claudélien, mérite qu'on s'y arrête un instant, dans la mesure où, précisément, il déborde le cadre de la poésie. Car ce poète est aussi un dramaturge dont certaines œuvres ont connu un énorme succès. Son biographe, René Pageau, dans sa thèse intitulée Gustave Lamarche poète dramatique, indique que La Défaite de l'Enfer, « jeu choral évangélique de sept cents figurants, joué dans la montagne de Rigaud » a été créé en 1938 et que «à Ottawa, en 1947, on jouait Notre-Dame de la Couronne [...] devant cent mille spectateurs $^{6}$. » Reste à savoir comment interpréter un tel succès populaire par rapport à l'influence proprement dite du théâtre claudélien, à la fois du point de vue dramaturgique et de l'esprit religieux. 
Quoi qu'il en soit, comme le révèle l'exemple de Gustave Lamarche, on peut parler d'une période faste, de 1928 jusqu'aux environs de 1950, au cours de laquelle Claudel domine en maître, même s'il n'échappe pas, ici et là, à une certaine contestation, (Lebel parle de deux clans : pro-claudéliens et anti-claudéliens) et s'impose (il conviendrait peut-être mieux de dire qu'on l'impose) comme le modèle idéal, celui du poète catholique dont le Canada français n'a pas d'équivalent, bien qu'il s'efforce de faire naître un mouvement national capable de promouvoir son propre théâtre à un rang qui lui permettrait de rivaliser sans complexe avec les grands auteurs français. Telle est l'ambition avouée de Gustave Lamarche qui, tout en admirant l'auteur de La Trilogie, de l'Annonce faite à Marie, du Soulier de satin, s'efforce de promouvoir une littérature spécifiquement québécoise, non esclave du seul modèle français que l'on reconnaît en Claudel.

Pour G. Lamarche, il ne suffit pas, dans cette perspective, de se faire théoricien. Il importe de donner l'exemple, et, confiant en lui-même, il compose des pièces selon l'esprit de ce qu'il considère comme le meilleur théâtre, celui qu'illustrent Henri Ghéon et Claudel, c'est-à-dire des pièces spirituelles, populaires, destinées au grand nombre et non à une élite intellectuelle, et illustrant des valeurs universelles. Certes, Gustave Lamarche n'a pas la prétention, du point de vue dramaturgique, de rivaliser avec Claudel. S'il ne cache pas qu'il recherche dans l'art claudélien « des inspirations de forme et de fond », il reconnaît que cet art, bien que génial, est «parfois obscur et complexe dans ses intrigues et son expression ?. » Ce qui, implicitement, fait un nombre de défauts propres à inquiéter, voire à décourager, plus d'un spectateur, et plus encore tout éventuel imitateur de Claudel. Mais, pour sa part, G. Lamarche a su percevoir chez ce dernier, comme chez H. Ghéon, l'importance de l'élément comique, de l'humour, de la liberté prise par ces auteurs avec le respect généralement accordé au sacré. Cependant, tout en recourant lui-même au comique, il ne se décide pas à traiter d'autres sujets que bibliques, à quelques rares exceptions près. Claudel, lui, bien que très marqué par la lecture de 


\section{Bernard Hue}

la Bible, n'emprunte pas les sujets de ses grands drames, même celui de L'Annonce... au Livre sacré; ce n'est qu'après Le Soulier de satin, lorsqu'il se consacre presque exclusivement à l'exégèse biblique, qu'il compose des oratorios dont les sujets et les personnages sont directement empruntés à la Bible.

Autre différence à souligner : le théâtre de Claudel doit beaucoup à l'expérience personnelle du poète qui ne cache pas que la plupart de ses personnages, issus de lui-même, sont à son image. Dans son théâtre, le conflit majeur réside dans la lutte du chrétien, partagé entre le péché et l'interdit religieux. Et la mise en scène de cette lutte n'a rien d'un sermon, ni d'un commentaire biblique. Loin d'éclairer une situation dramatique complexe, tragique, Claudel la rend souvent ambiguë, mystérieuse, difficile à expliquer même pour un chrétien.

G. Lamarche, lui, en s'en tenant presque exclusivement aux seules données bibliques, prend une énorme distance par rapport à celui qu'il s'est donné comme exemple. Et même lorsqu'il se risque à traiter un drame moderne, il serait aisé de montrer en quoi l'auteur québécois diffère profondément de Claudel. Par exemple, l'analyse de La loi du feu (1948), dont le sujet rappelle ceux de l'Annonce... et du Père humilié, révèle davantage une volonté de prosélytisme de la part du religieux que de profondeur humaine chez ses héros. Lull, en effet, n’hésite pas à sacrifier sa bien-aimée Loyse et son amour pour elle, afin de courir à l'aide de son frère Prince avec lequel il a toujours entretenu une parfaite entente. Et son dévouement, son sacrifice, recevront de Dieu une récompense digne, aux yeux de l'auteur, du devoir qui n'a pas entraîné le chrétien dans des tourments comparables à ceux des héros claudéliens en proie au péché, au remords, allant à l'occasion, comme Mesa, jusqu'au sacrilège, jusqu'à se révolter contre Dieu. Humanité et sainteté ne cessent de torturer les héros dont Claudel met en scène la difficile soumission à l'ordre religieux.

Pure coïncidence, si ce n'est influence : en 1968, Gustave Lamarche fait paraître, à Montréal, Le Conte des sept jours, deux ans après la publication posthume $\mathrm{du}$ grand commentaire biblique de Claudel, Au milieu des vitraux de l'Apocalypse, qui était en cours 
d'écriture en 1929 et constitue le premier état probable de Paul Claudel interroge l'Apocalypse. Son chapitre IX porte l'indication "Octobre 1930 » et évoque explicitement le second voyage au Canada. Ce chapitre commence ainsi :

J'en étais à l'Ennui, quand j'ai dû arrêter ma lettre pour monter dans le char de Pharaon et pour répondre à l'invitation qui m'était adressée de peindre l'automne entre la Susquehannah et le Saint-Laurent sur un châssis aussi large que la moitié de l'Europe. Les lacs sont comme les godets où l'on va rafraîchir son pinceau de temps en temps $^{8}$.

Excluons l'idée d'influence directe, purement hypothétique. Ce qui importe dans le rapprochement des deux œuvres religieuses, c'est la manière commune aux deux poètes d'aborder et de traiter le livre sacré. Et ce qui caractérise cette manière tient essentiellement en deux points : l'interprétation figurative, c'est-àdire anti-littéraliste, comme l'explique Claudel dans son Introduction au Liore de Ruth, intitulée " Du sens figuré de l'Écriture ", et la liberté avec laquelle les deux poètes, se mettant en scène, disant « je », faisant état de leurs sentiments, de leurs souvenirs, de leurs préventions, de leur expérience personnelle, privilégiant l'humour, évitant toute distanciation entre eux et la Bible, parlent, l'un de l'Apocalypse, l'autre de la Genèse, le religieux n'hésitant pas à présenter son commentaire de « l'Annale infaillible ${ }^{9} "$, comme un simple conte. Irrespect? Plaisanterie? Il s'en faut. Il ne s'agit pas d'une entreprise de désacralisation qui aurait quelque chose de sacrilège. Dans les deux cas, l'art de séduire, de plaire, de rendre le message aisément ou relativement accessible, l'emportent sur la technique traditionnelle d'une laborieuse exégèse, savante, pleine de gravité, non à la portée d'un lecteur dépourvu de sérieuses connaissances théologiques. D'une façon générale, Claudel a horreur des pédants. Il ne ménage pas son ironie contre eux dans Le Soulier de satin, et dans le commentaire de 1928, il écrit : 


\section{Bernard Hue}

Qu'est-ce que nous sommes, nous autres, pauvres petits catholiques, à côté des Géants de la Science et de la Philosophie Modernes (on ne mettra jamais trop de majuscules) ${ }^{10}$.

Il préfère à l'explication (sans doute trop cartésienne à ses yeux) la technique de la promenade allégorique, symbolique. À sa fille qui lui avoue n'avoir rien compris au livre de l'Apocalypse, le père répond péremptoirement en pédagogue peu conformiste :

Il ne s'agit pas de comprendre l'Apocalypse mais de se promener dedans comme des ignorants qui, entrant pour la première fois dans une cathédrale, découvrent un spectacle (office en latin) étonnamment beau bien que le sens leur échappe. L'image doit l'emporter sur le concept pur. Le commentaire doit être une figuration, et faire appel constamment à la poésie ${ }^{11}$.

Gustave Lamarche ne procède pas autrement en présentant le Livre de la Genèse comme une parabole, un conte, un « conte vrai ", un drame ${ }^{12}$, qui lui permettent de grandes libertés (jeux de mots, humour, ironie, dérision). Adam et Eve sont appelés les deux Amants, les chers Amants, Isch et Ischah. Comme Claudel, le poète québécois aime les anachronismes, les plaisanteries avec le temps. "Isch aime beaucoup, comme saint François, parler aux oiseaux $^{13}$ "Très à l'aise avec la Bible, G. Lamarche se rencontre, dans son esprit et dans les procédés narratifs qui agrémentent son commentaire poétique, avec Claudel dont les audaces de langage, la force des images (satirique, à l'occasion), la dérision (il qualifie sa démarche de "sapience interprétative » ${ }^{14}$ ), mêlée à l'admiration issue d'une conviction spirituelle inébranlable, font de son exégèse une réécriture, un poème, beaucoup plus qu'une élucidation intellectuelle d'un texte que des siècles d'examens, de gloses, de commentaires ont privé de toute force de nouveauté et, en apparence, de pouvoir d'inspiration. 
Le Conte des sept jours est parcouru de nombreuses réminiscences claudéliennes : tête d'or, repos du Créateur le Septième Jour, le mal, personnification de la Sagesse (assimilée à la Vierge Marie) ${ }^{15}$. La même influence se retrouve dans un autre ouvrage d'inspiration foncièrement religieuse, Le Beau Dieu, publié en 1975, dont la nature abstraite est annoncée par le sous-titre : ou trentetrois clameurs de l'âme. Cette fois, le but avoué de G. Lamarche est d'orienter son commentaire vers une connaissance. Perspective pédagogique sans rapport avec le jeu dramatique, mais non sans parenté avec l'exégèse biblique pratiquée par Claudel. Tout se passe comme si le religieux québécois avait été conduit, fût-ce malgré lui, à demeurer tributaire du grand modèle claudélien. Quand il écrit Le Beau Dieu, G. Lamarche se fait très pédagogue, frôle le sermon, interpelle ses lecteurs : « frères », «bien-aimés » ${ }^{14}$ et fait une dissertation plutôt que de se livrer à un commentaire très libre, à la manière de Claudel. En réalité, le poète québécois imite l'esprit du drame de Claudel, et, s'il atteint le grand art religieux dans l'exégèse biblique, il est loin d'égaler les hautes réalisations scéniques qui ont assuré le succès du poète français, éreinté parfois, par des compatriotes catholiques qui ont déploré dans ses drames la contradiction, l'inadéquation, entre la rigueur de la tradition, de la loi religieuse et la liberté humaine (le désir de la créature humaine érigé en loi). Thêâtre non-conformiste privilégiant le conflit par excellence, et le pire, le conflit entre l'homme et Dieu. À l'inverse, le théâtre de Gustave Lamarche peut être vu comme le meilleur exemple de conformisme.

D'ailleurs, les années 1968-1975 ne sont plus guère favorables à une influence religieuse, d'où qu'elle vînt ${ }^{17}$. La fortune littéraire de Claudel, au Canada, s'estompe; le catholicisme ne domine plus seul la pensée, ni les attentes d'une société en pleine mutation, décidée à bousculer la tradition dans laquelle elle a été enfermée comme dans un carcan devenu insupportable. Depuis la seconde guerre mondiale, s'est dessiné peu à peu un mouvement de mise en question. N'ira-t-on pas jusqu'à s'interroger sur le bien fondé de l'admiration vouée à Claudel, en regardant ses héros avec 
un œil sinon moins bienveillant, du moins plus attentif à déceler ce qui fait d'eux des êtres non rigoureusement conformes à l'idée généralement répandue concernant les saints, êtres hors du commun, soumis, consentants, respectueux de la loi divine? Oser un tel regard, n'est-ce pas déjà une véritable entreprise de désacralisation?

C'est déjà ce qui est en train de se réaliser dans les années de la seconde guerre mondiale et de l'après guerre. Et, bien que peu prévisible, la plus manifeste expression de ce bouleversement apparaît chez une jeune Québécoise, Anne Hébert, à peine connue en 1940. Une authentique filiation se dessine entre elle et Claudel, sans qu'on puisse accuser la jeune lectrice du poète français de faire preuve d'une soumission proche de la servilité. Certes, ayant reçu une importante initiation biblique, Anne Hébert était préparée à accueillir l'œuvre de Claudel. D'ailleurs, elle reconnaît la place occupée par ce dernier dans sa formation littéraire. Et elle ne rejette pas le catholicisme qui imprègne aussi bien l'œuvre de Claudel que la culture et l'éducation nord-américaines qui ont présidé à sa formation. Quand, en 1980, Donald Smith lui dit : « Je sais, par exemple, que vous avez été fortement impressionnée par Claudel ", et lui demande si elle se percevait alors comme un écrivain catholique, elle apporte cette précision :

J'étais très catholique comme tout le monde au pays à cette époque-là. J'étais profondément religieuse et croyante. C'était donc normal que j'en parle. Cela faisait partie du réel quotidien ${ }^{28}$.

Si le catholicisme lui est apparu, au temps de sa jeunesse, comme une cause de souffrance, - Donald Smith va jusqu'à parler d'une "sorte de crucifixion »-Anne Hébert se plaît à souligner que ce fut aussi une source de richesse :

Oui, c'est une richesse aussi, et je crois que les Québécois aujourd'hui qui ont coupé complètement avec la religion se coupent en même temps de toute une richesse, qui est 


\section{Imitation et désacralisation de Claudel au Canada français}

la richesse de la Bible. Pour moi, ç'a été très contraignant, mais en même temps une richesse.

On ne sera donc pas surpris de rencontrer chez Anne Hébert, dès ses premières œuvres, non seulement une parenté dans les thèmes, mais un certain nombre d'emprunts, parfois de véritables calques, provenant de ses lectures du poète français. Elle se plaît même à en signaler si, le cas échéant, on ne percevait pas clairement ce phénomène d'intertextualité. Ainsi, elle signale à Neil Bishop une phrase du Premier Jardin, qu'elle a pourtant pris soin de souligner dans la graphie même du roman, empruntée au poème «Pensée en mer » de Connaissance de l'Est : « La séparation a eu lieu, et l'exil où il est entré le suit ${ }^{19}$. » Cet exemple, à lui seul, suffirait à révéler la parenté entre les deux auteurs. Le thème de l'exil étant au cour du roman de la Québécoise, celle-ci trouve d'emblée, chez Claudel, un lien avec sa propre pensée. En n'hésitant pas à mettre en évidence un emprunt textuel ouvertement affiché, elle affirme ce lien qui ne se réduit pas à une simple rencontre d'ordre religieux, le poème de Claudel, en l'occurrence, relevant avant tout de la confidence biographique.

Une étude des thèmes majeurs développés par l'ensemble de l'œuvre d'Anne Hébert ferait apparaître un parallélisme souvent saisissant, en particulier entre les drames de Claudel et les romans d'Anne Hébert. Qu'on pense, par exemple, non seulement à la notion d'exil, mais au péché, à la violence, à l'opposition entre la lumière et l'obscurité, au désir et à ses contradictions, aux interdits d'ordre religieux, aux conséquences tragiques de toute transgression.

Tenons-nous-en à l'exemple de la pièce la plus populaire de Claudel, L'Annonce faite à Marie, qui a inspiré à Anne Hébert sa première grande œuvre théâtrale, L'Arche de midi, composée en 1944-45, mystérieusement demeurée inédite, et portant un titre propre à créer une confusion avec une autre production claudélienne, Partage de midi. Parallèlement, paraît, en janvier 1945, un article d'Anne Hébert, intitulé L'Annonce faite à Marie, à propos duquel on peut se demander s'il visait à préparer une éventuelle 
publication de L'Arche de midi, réplique très libre de la pièce française, au moment même où Claudel, dont Jean-Louis Barrault vient de créer Le Soulier de $\operatorname{satin}^{20}$, reprend pour la cinquième fois son texte de L'Annonce (dite version pour la scène). Anne Hébert ignore, naturellement, que la pièce qu'elle a entre les mains ne constitue pas le drame définitif qu'elle entend réécrire. Toujours estil qu'elle offre au public québécois, par l'intermédiaire de la Reque dominicaine, dirigée par Antonin Lamarche, O. P. une vision originale de la pièce de Claudel (dont elle ne mentionne aucune fois le nom, tant le titre de l'œuvre, à ses yeux, suffit à le désigner). Cette vision permet de deviner ce qui, dans ce drame du miracle et de la sainteté, frappe et peut-être offusque la jeune dramaturge décidée à secouer le joug qu'impose à la femme québécoise une société encore dominée par la tradition patriarcale mise précisément en valeur, avec quel relief! dans la pièce « médiévale » de Claudel.

L'article d'Anne Hébert, plus proche, par le style et la structure, d'un poème en prose que d'un examen rigoureusement critique, devrait être analysé mot à mot. De cette analyse ressortiraient les données suivantes :

Une grande réserve faisant place à une objectivité qui évite à l'auteure de dire je et de porter explicitement un jugement personnel.

Une certaine obscurité due à la métaphorisation de l'exposé, critique et objet de la critique recourant à un même style.

Un non-dit quelque peu réducteur par souci de simplification et de mise en relief de l'essentiel (amputant des aspects de la pièce, en particulier psychologiques).

De ce premier constat, se dégage l'impression que l'auteure manifeste un sentiment ambigu à l'égard de la pièce de Claudel qu'elle présente d'emblée en ces termes à la fois exacts et réducteurs :

« L'Annonce faite à Marie » c'est le drame de la sainteté.

Et, consciente de l'équivoque recelée par cette définition (de quel drame s'agit-il : de celui qu'elle vient de nommer, L'Annonce..., qui 
a pour sujet la sainteté, ou du drame que constitue en soi la sainteté ?) elle s'empresse de définir cette délicate notion d'ordre strictement religieux en renvoyant habilement au commentaire du dramaturge dans lequel la qualité, le concept d'ordre spirituel, est remplacé par la figure humaine de l'être défini par la qualité d'où est tirée son identité :

Claudel a écrit ailleurs que « le saint est celui que Dieu ne laisse pas tranquille $»$.

À la manière d'un syllogisme, suit la conclusion, qui a la simplicité d'une évidence :

Violaine est quelqu'un que Dieu ne laisse pas tranquille.

Plus loin, Anne Hébert présentera Mara, figure antithétique de Violaine, comme celle «que le diable ne laissait pas tranquille ", soulignant le manichéisme de la pièce de Claudel.

Donc Dieu dérange, importune la jeune héroïne. Et il est mis sur le même plan que les hommes, Anne Hébert le présentant même comme le rival de Pierre de Craon et de Jacques Hury, hommes de désir. Dieu se trouve ainsi ramené au niveau de sa créature, ce qui, de la part d'Anne Hébert, est faire preuve d'une audace quasi sacrilège. De plus, ne s'en tenant pas à son rôle de critique, elle n'hésite pas à recourir à une métaphore d'une grande violence pour rendre plus saisissante l'action divine :

Et voilà que Dieu fond sur elle comme un aigle.

L'image n'étant pas neuve, on ne saurait prétendre qu'Anne Hébert l'ait empruntée à Claudel qui, en 1932, écrivait :

[...] il fond sur nous comme un aigle pour reprendre appui sur le sol $[\ldots]^{21}$ 


\title{
Bernard Hue
}

Cette image trouvera place à la fin du premier acte de L'Arche de midi, dans la bouche de Pierre contemplant Marie endormie :

\begin{abstract}
Je suis rude, mais je suis patient ${ }^{22}$. [...] Parfois il faut être prompt et fondre sur la proie comme un hibou, bien caché dans la nuit, tenu par le seul vol de ses ailes silencieuses et de son désir précis. (acte I, scène IX)
\end{abstract}

Comment, à propos de L'Annonce, présenter plus clairement l'équation dans laquelle la sainte, Violaine, est synonyme de victime innocente? Et face à l'innocence menacée, Anne Hébert développe le thème du désir, terme-clé qui revient à quatre reprises dans quatre phrases successives. Il en ira de même du thème de la Voix, mot qui apparaît cinq fois en six phrases brèves. Le désir de Dieu est souligné par l'anaphore dans laquelle le mot désir, déterminé par un nom introduit par la préposition sur, constitue une image faite, semble-t-il, pour coïncider avec l'expression « fondre sur »: «Le désir de Dieu sur Violaine ». À ce désir répond le oui spontané de Violaine. Pour elle, tout est simple; il est vrai, assure Anne Hébert, (est-ce sans quelque ironie?), qu'elle a le privilège de n'avoir rien à décider :

La Voix fait elle-même les demandes et les réponses, la proposition et l'exécution.

C'est ainsi que Violaine, mue par la charité, consent immédiatement dans l'allégresse à ce qui lui est imparti par le truchement de la voix surnaturelle. Ensuite se feront jour les effets de ce consentement forcé : la lèpre et ses conséquences. Le drame correspond au déroulement du Sacrifice, semblable au « jeu de la Messe ».

Suit la réaction des êtres déçus, qui se croient tous trahis, sacrifiés par Violaine : tous l'abandonnent, la relèguent dans l'obscurité et le silence. La douleur, allégorie développée dans une 
longue phrase au cours de laquelle ce mot se répète cinq fois, voilà le seul appui terrestre qui s'offre à Violaine condamnée au silence. Seul lui reste fidèle Dieu qui, finalement, a réussi à se substituer à sa victime : le Christ existe à la place de Violaine. Alors peut se produire le miracle. Violaine passe de l'obscurité à la lumière. Sa vraie nature, - sa sainteté -, est révélée au monde. Et Anne Hébert en vient à cette conclusion qui laisse percer, discrètement, son sentiment à l'égard de l'auteur de ce « jeu » liturgique :

\section{Le poète a tiré de son côté Violaine, cette Ève de grâce et de douleur, que nous avons reçue comme une sainte et comme un Message.}

Cet article, comme le suggère le mot de la conclusion : « Le poète a tiré de son côté Violaine », peut donc, en définitive, être interprété comme un procès dans lequel sont convoqués Dieu et les hommes dont le poète tire ce qui convient à sa création dramatique. Dieu, selon Anne Hébert, est présenté comme un rapace et la femme innocente comme la proie de ce prédateur. Dans un texte poétique, dont les versets font écho à ceux de Claudel, Anne Hébert met habilement en relief la faiblesse de l'homme en face de l'absolue volonté de Dieu, cette terrible volonté qui écrase la créature la plus chrétiennement soumise. Pouvait-elle, devant pareille tragédie ne pas s'élever contre le point de vue de Claudel qui, au nom de la religion, glorifie le sacrifice et le consentement de la femme destinée, si telle est la volonté de Dieu, à l'humiliation, à la violence, à l'abandon, à la mort? Et n'est-ce pas pour manifester son point de vue personnel, son désaccord par rapport à l'œuvre de Claudel, qu'elle écrit une autre Annonce, dans laquelle Marie tient le rôle de Violaine et où la mère porte le nom d'Elisabeth, comme l'épouse d'Anne Vercors? Les préoccupations idéologiques d'Anne Hébert, dès ses premières compositions, conduisent à se demander ce qu'elle a, ou aurait ressenti en lisant, dans la toute première version de L'Annonce..., ces mots que Violaine adresse à Dieu à l'heure de la mort dont sa sœur est la principale responsable : 


\section{Bernard Hue}

Regarde, voilà leur amour.

Mon père m'a abandonnée, ma mère, livrée; et mon fiancé M'a reniée, et ma sœur ${ }^{23}$...

Terrible bilan qui préfigure la situation de Marie dans L'Arche de midi, où Elisabeth-la-Complaisante, sa mère, force de destruction, l'offre à Pierre, un de ses anciens amants, et où le Boiteux, « dernier servant du culte d'Elisabeth ", resté son admirateur inconditionnel alors qu'elle s'est toujours refusée à lui, tuera l'adolescente à l'heure où celle-ci réussira à quitter l'espèce de cloître maléfique, la cabane de bois dans la montagne, dont, fidèle serviteur de « la vieille belle », il s'est fait le gardien, interdisant à Marie de s'en éloigner, toujours prêt à la frapper au moindre signe d'insoumission.

Dans son étude consacrée à l'Arche de Midi, Pierre-H. Lemieux, qualifiant cette oeuvre de superbe pièce, considère qu'Anne Hébert se dégage alors de « l'étreinte pseudo-catholique comme de l'influence claudélienne ${ }^{24}$. "Qu'elle entreprenne de se libérer et de libérer ses jeunes compatriotes, du carcan dans lequel leur éducation religieuse les tient encore enfermés ne fait aucun doute. Quant à l'influence claudélienne, il s'agit non d'un rejet du modèle esthétique, mais d'une influence par réaction, celle-ci intéressant la conception archaïque de la société telle que Claudel s'est plu à la découvrir au Canada.

Anne Hébert est manifestement sensible à l'art du poète français ${ }^{25}$. En 1942, elle écrit deux poèmes : Ève, publié dans la revue Amérique française, et Éveil au seuil d'une fontaine. La fontaine jouera un rôle important dans L'Arche, où Marie, après avoir trouvé un sens à sa vie, sera tuée par le Boiteux. On observera surtout qu'entre 1938 et 1945, Anne Hébert produit cinq nouvelles, dont «Le Torrent ». Le thème principal de cette nouvelle qui donnera son titre au recueil publié en 1950, mérite, compte tenu de ses accents claudéliens, d'être mis en parallèle avec celui de L'Arche, composée en 1944-45, «Le Torrent " datant lui aussi de 1945. Yves Préfontaine devait dire avoir été vivement impressionné par cet « extraordinaire recueil de nouvelles, d'une belle 
étrangeté et parfois, d'une dureté surprenante ${ }^{26}$. »C'est, en effet, la violence et la cruauté qui frappent dans Le Torrent, comme elles frappent dans le drame demeuré inédit, inspiré par le drame le plus ouvertement religieux de Claudel. On ne saurait donc être étonné de découvrir, parmi les thèmes majeurs des nouvelles, des affinités avec certains thèmes claudéliens. On remarquera, par exemple, la toute première phrase de la nouvelle éponyme :

J'étais un enfant dépossédé du monde.

Le thème de la dépossession convient parfaitement pour définir aussi ce qui advient à Violaine à laquelle tout est ravi, qui est chassée de chez ses parents, éloignée de son village, lépreuse vivant comme une étrangère privée de toute identité, obligée de trouver un refuge dans une caverne et qui, finalement, perd la vie par la volonté malfaisante de sa soeur. Le Torrent relate, comme le précise Anne Hébert, « l'histoire d'un fils qui est démoli par sa mère ${ }^{27}$. "Ce fils, François Perrault, constitue, avec la grande Claudine, sa mère possessive et brutale, l'équivalent de la jeune Marie et d'Elisabeth, mises en scène dans L'Arche, Marie, comme François, étant enfant du péché. Et le vœu de François, « Je voudrais croire en Dieu », semble pouvoir être perçu comme l'expression explicite de ce que recherche Marie, la nuit, lorsque sa marche initiatique la conduit à la fontaine. Quête de leur identité, mais aussi quête d'une vérité permettant aux deux héros de tourner le dos au péché et de réussir, par leur seule force, à échapper au mal. Inversion de la situation de Violaine qui, elle, bien insérée dans une famille chrétienne, bénéficie d'une vie heureuse dans son enfance, jusqu'au jour où, par la volonté de son père, elle devra consentir à épouser l'homme que ce dernier lui impose et qui semble convenir parfaitement à la jeune fille. Comme dans un conte, le début de la pièce de Claudel, présente un monde harmonieusement organisé, où tout est pour le mieux. Mais, nous l'avons vu, Dieu veille jalousement sur sa proie. Chez Anne Hébert, à l'inverse, l'innocent est seul, rejeté parmi des marginaux, contraint de chercher à tâtons le chemin de la grâce pour échapper 


\section{Bernard Hue}

au destin maléfique dans lequel veut l'enfermer une mère à demi folle, chassée du village où elle a été vue comme objet de scandale, enfermée dans son passé, refusant l'idée de vieillissement. Ce lieu symbolise l'enfer d'où Marie croira pouvoir s'enfuir, avec l'aide et l'amour de Pierre. Mais pour elle, la libération espérée n'aura été qu'une tragique illusion. Elle meurt assassinée, comme Violaine.

Or, Anne Hébert supprime de L'Arche de midi le personnage de Mara et privilégie celui de la mère, Elisabeth-la-Complaisante, tout en mettant au cœur du drame Marie, l'enfant sacrifiée par une mère qui fait de ce sacrifice le seul moyen de voir toujours venir chez elle des hommes dont elle veut croire, dans sa folie, que c'est par fidélité, par amour pour elle qu'ils continueront à fréquenter sa cabane, annoncée comme lieu de malédiction par le Témoin ${ }^{28}$. L'auteure privilégie cette question de la femme aux prises avec une société où domine le mal, vivant en marge des valeurs morales traditionnelles, sans souci de la religion catholique. Audace dans la transgression opérée par Anne Hébert. Présage d'œuvres à venir, où les héroïnes feront souvent fi des interdits socio-religieux. Le sacrifice de Violaine peut sembler scandaleux à Anne Hébert qui, dans son essai, le présente comme la volonté expresse de Dieu, en quelque sorte substituée, dans L'Arche, à la volonté de Mara.

Toute l'œuvre d'Anne Hébert permet, a posteriori, de comprendre ce que, dès sa jeunesse, la romancière a pu, implicitement, reprocher à Claudel. Dans L'Annonce, celui-ci, se réfugiant dans un moyen âge fort conventionnel, met en scène des personnages très modernes, repris au premier état de son drame, La Jeune Fille Violaine (1892), où l'inquiétant Pierre de Craon n'existe pas encore. Éloge de la famille patriarcale, de l'ordre social dominé par la religion, que Claudel maintient désormais jusque dans l'ultime version (1946). Cet éloge des vertus qui confinent la femme dans une sorte de réclusion que lui aurait valu le péché originel ne saurait être approuvé par Anne Hébert qui ne peut que se révolter devant le sacrifice de la jeune fille privée de tout choix personnel, de tout désir, de toute liberté, ainsi que devant le silence coupable de la mère, Elisabeth, qui cède au chantage diabolique de Mara, jalouse de sa sœur. D'où l'énigmatique mot de la première version 
de La jeune fille Violaine : "Mon père m'a abandonnée, ma mère, livrée... ", qui a pour écho, mais dans une tout autre perspective, l'amer souvenir qu'évoque Marie quand, au début de L'Arche, sa mère lui parle du médaillon que lui a donné Pierre :

\begin{abstract}
(D'une voix tremblante) Tu sais bien que Pierre est comme un mort. (Très bas). Il n'est pas venu ici depuis l'année dernière, ce soir d'été plein de pluie et d'odeurs où tu m'as mise en son lit pour dormir... (acte $\mathrm{I}$, scène IV)
\end{abstract}

En écrivant L'Arche, Anne Hébert entend traiter le problème fondamental de l'enfance étouffée par le milieu familial dans lequel la mère (image d'Ève) est coupable, ou, conformément au code traditionnellement admis et maintenu par l'Église, est réputée coupable. Quelle est sa faute? D'être femme et d'avoir osé aimer, librement, jusqu'à transgresser toute loi, jusqu'à en perdre la raison. Au fond, l'idée directrice qui conduit Anne Hébert à écrire des œuvres d'une rare violence, est celle que développe Partage de midi, pièce autobiographique dans laquelle Claudel célèbre, d'une manière violemment païenne, la passion amoureuse illicite qui lie Ysé, la mère coupable, à Mesa, le chrétien qui élève la voix contre Dieu, dans un scandaleux défi. Dans L'Arche, où passe quelque réminiscence de Partage de midi, Pierre invite Marie à consentir à sa condition de femme, à sortir de « ce monde crucifié " :

Marie, Marie, le temps presse, acceptons d'être chassés de l'Éden, une fois pour toutes, ensemble, l'un par l'autre, unis par même péché et semblable nostalgie de l'arbre entamé. Je serai à toi et tu seras à moi. (acte III, scène VII)

Pour cela, après de secrètes errances nocturnes, il faudra franchir un pont fragile, « la haute porte de Midi », « la plus profonde porte, l'Arche la plus singulière, sous la flamme tranquille de midi », flamme qui devrait combler le " grand vide de midi " dont 


\section{Bernard Hue}

il sera question dans Poèmes ${ }^{29}$. Marie meurt au moment même où, rejoignant Pierre, elle allait réaliser leur rêve commun. Une dernière fois, revient l'image obsédante du rapace associée à une question angoissée et peu orthodoxe du héros :

Marie, frêle appeau, devenue proie abattue, Dieu est-il le complice secret de ma chasse?

On est loin, avec ce théâtre d'Anne Hébert, de l'entreprise de Gustave Lamarche. Si l'un et l'autre ont une dette à l'égard de Claudel, celle-ci diffère selon qu'on a affaire aux pièces bibliques du religieux destinées à maintenir un ordre ancestral établi sur des valeurs strictement religieuses, considérées comme non susceptibles du moindre écart, ou à la production poétique et dramatique d'Anne Hébert qui, tout en acceptant la manière symboliste dans laquelle s'épanouit le génie et s'exprime le style de Claudel, n'hésite pas à prendre du recul par rapport aux idées du poète français en harmonie avec celles des Québécois conservateurs à la manière de Gustave Lamarche, convaincue que ces idées ont conduit son pays à une aliénation sociale, en faisant de la condition féminine, en particulier, une condition quasi carcérale. Claudel, au Canada, était loin de pouvoir prévoir le mouvement d'émancipation, la libération qui allaient ne pas tarder à s'y faire jour et qui ébranleraient ce qu'il considérait, en 1937, comme une assise inébranlable qui lui faisait dire :

Depuis [1928-1929], ma pensée se reporte bien souvent à cette terre puissante où les vertus antiques ont pris de nouvelles racines et où la famille, sans desserrer ses liens consolidés par l'épreuve, s'est élargie aux dimensions d'une patrie dont la charte est un sacrement.

Et il se flattait de jouir dans cette nouvelle France, d'après les échos qui lui en arrivait (en particulier par l'intermédiaire du cardinal Villeneuve), d'une vive sympathie qu'il attribuait essentiellement au catholique qu'il était : 
... je sais que l'on aime là-bas à écouter la voix d'un poète qui a puisé son inspiration [...] dans le chant des psaumes autour du lutrin de son village, dans la véhémence du prédicateur chrétien, dans la complainte du laboureur qui au travers d'une terre consacrée renouvelle le sillon de l'espérance, et dans le conseil de l'alouette ${ }^{30}$.

L'histoire permet, aujourd'hui, de faire la juste part des choses. Le culte de Claudel n'a pas été qu'un mythe né de l'admiration que pouvaient lui porter des catholiques voyant en lui le meilleur garant des valeurs qu'ils souhaitaient voir perpétuer dans un Canada célébré par le grand poète français dont, à leurs yeux, l'œuvre méritait d'être élevée au rang de modèle, faisant ainsi de Claudel un maître à penser, ce qui n'était pas le cas chez tous les catholiques français. Un tel modèle, dont l'œuvre, comme l'a bien senti Anne Hébert, n'était pas sans ambiguïtés, pouvait-il survivre à la profonde transformation des mentalités, réaliser le rêve de Gustave Lamarche? Pouvait-il aider les écrivains de la génération d'Anne Hébert à affirmer leur propre identité en secouant le joug d'une tradition sclérosante, que Claudel d'ailleurs, pour la rendre acceptable en France même, devait présenter dans ses grands drames, comme celle d'un temps très reculé, le XVI e siècle espagnol dans Le Soulier de satin, le moyen âge dans L'Annonce faite à Marie?

\section{Notes}

1 Voir "Paul Claudel au Canada Français » par Maurice Lebel, in Claudel Studies, Volume XI, 1984, p. 56-66.

2 «La chanson française », Pr., p. 380.

3 Ibid.

4 Pour son séjour à Toronto, voir Sergio Villani, « Paul Claudel à Toronto en 1928 , in Paul Claudel : Les Odes, Les Éditions Albion Press, 1994, p. 1-14.

5 Curieusement, ce constat est immédiatement assorti de cette remarque qui ne laisse pas de surprendre quant au peu d'intérêt suscité par l'œuvre de ce religieux québécois présentée comme la plus rede- 


\section{Bernard Hue}

vable au poète français : « dont les œuvres sont mieux connues et plus étudiées à l'Université de Haute-Bretagne, à Rennes, que dans les Facultés de Lettres du Canada français. "

6 Gustave Lamarche poète dramatique, Les Éditions Garneau, 1976, p. 195.

7 Cité par René Pageau, Le Père Gustave Lamarche, poète dramatique, Thèse dactylographiée, 1974, Université de Haute-Bretagne, Rennes II, p. 282, 227.

8 Au milieu des vitraux de l'Apocalypse, Gallimard, 1966, p. 171.

9 Le Conte des sept jours, Éditions de la Parabole, Montréal, 1968, p. 123.

10 Au milieu des vitraux de l'Apocalypse, p 183.

11 Id., p. 11.

12 Le Conte des sept jours, p. 98, 121.

13 Id., p. 127.

14 Id., p. 156.

15 Id., p. 114, 124, 152, 153, 212 n.

16 Le Beau Dieu, Éditions Pleins Bords, 1975, p. 97, 13.

17 Répondant à une lettre dans laquelle je lui parlais de la situation actuelle de Claudel dans l'enseignement supérieur en France, Gustave Lamarche m'écrivait, en 1975 : «Vous me demandez si la situation est meilleure au Québec pour les ouvrages de mon «Beau Dieu ». Hélas non : On cherche ailleurs la " beauté ". Et je ne suis pas trop sûr qu'on la trouve. La télévision se charge d'alimenter le peuple québécois en choses bonnes et belles. Je préfère ne pas vous dire quelles sortes de faims trouvent là leur rassasiement... »

18 Lettres Québécoises, Hiver 80-81, n²0, p. 67.

19 In Études Canadiennes, A.F.E.C. 1990,28 , p. 37 et 56.

$20 \mathrm{La}$ fonction du personnage appelé Le Témoin dans L'Arche de midi rappelle celle de l'Annoncier dans Le Soulier de satin. Publié en 1928, ce drame ne pouvait pas avoir échappé à l'attention du père d'Anne Hébert, critique littéraire très au courant de l'actualité littéraire en France. Il serait intéressant de connaître le contenu de sa bibliothèque personnelle.

21 Cité par Marie-Joséphine Whitaker in L'écriture de l'exégèse dans l'œuvre de Claudel, Presses universitaires de Franche-Comté, 2006, p. 45. 
22 À propos de L'Annonce, Claudel devait dire : « Le royaume de Dieu souffre violence ». Cette phrase pourrait servir d'exergue à ma pièce. «La forme la plus dangereuse de violence est la patience. » (Théâtre, Pléiade, tome II, p. 1396)

23 La jeune fille Violaine, Théâtre, Pléiade, tome I, p. 552. Anne Hébert réserve pour Le temps sauvage le thème des deux sœurs dont l'une, jalouse, réussira à s'emparer de l'amant de l'autre.

24 P.-H. Lemieux, Le Thêâtre canadien-français, éditions FIDES, Montréal, 1976, Archives des Lettres canadiennes, tome V, p. 555.

25 Gilles Lamontagne indique, à propos de Poèmes, recueil publié en 1960, qu'on a souvent remarqué que « les versets des quinze textes qui composent Mystère de la parole se situent, du point de vue formel, dans la tradition des grandes odes de Paul Claudel et de Saint-John Perse. » Dictionnaire des CEuvres littéraires du Québec, IV, 1960-1969, p. 690.

26 Y. Préfontaine, «La poésie d'Anne Hébert : l'ancrage québécois d'une esthétique de la colère ", in Anne Hébert, parcours d'une cuure, L'Hexagone, 1997, p. 51. - Anne Hébert précise à Donald Smith qui évoque les difficultés pour les femmes de se faire publier au Québec dans les années 1950: «A cette époque-là, c'était très difficile de publier par exemple Le torrent, parce qu'on le trouvait trop violent ", "Anne Hébert et les eaux troubles de l'imaginaire ", in Lettres Québécoises, Hiver 80-81, n²0, p. 66.

27 Cité par Lise Gauvin, "Les nouvelles du Torrent, un art d'échos ", in Anne Hébert, parcours d'une oeuvre, L'Hexagone, 1997, p. 221.

28 Sur L'Arche de midi, voir : - Neil Bishop, "Guerre, errances et exils dans l'œuvre d'Anne Hébert », in Anne Hébert, parcours d'une œuvre, l'Hexagone, 1997, les pages 165-167 sont consacrées à L'Arche de midi; - Bernard Hue, "Claudel revu par Anne Hébert, L'Arche de midi, réécriture de L'Annonce faite à Marie », in Imaginaires francophones, Publications de la Faculté des Lettres Arts et Sciences humaines de Nice, $\mathrm{N}^{\circ} 22,1995$, p. 207-215.

29 Voir Lucille Roy, « Les jeux de lumière et d'eau dans l'univers poétique d'Anne Hébert ", in Anne Hébert, parcours d'une oeuvre, l'Hexagone, 1997, p. 39. - Thème foncièrement claudélien, le jeu de la lumière et des ténèbres est également récurrent chez Anne Hébert. Voir pour ce qui concerne Claudel, Alain Vercollier, « Le Jour et la nuit dans les Cinq Grandes Odes », in Paul Claudel : les Odes, Les Éditions Albion, 1994, p. 89-100. 